\title{
The Inertia of Light and the Isotropic and Anisotropic Properties of Electro-magnetic Mass
}

\author{
Wim Vegt \\ Department of Physics, Technical University Eindhoven, Eindhoven, The Netherlands \\ Email address: \\ wimvegt@topacademy.center

\section{To cite this article:} \\ Wim Vegt. The Inertia of Light and the Isotropic and Anisotropic Properties of Electro-magnetic Mass. American Journal of Astronomy and \\ Astrophysics. Special Issue: The Interaction Between Gravity and Light. Vol. 7, No. 2, 2019, pp. 18-32. \\ doi: 10.11648/j.ajaa.20190702.11
}

Received: August 6, 2019; Accepted: September 16, 2019; Published: September 29, 2019

\begin{abstract}
Photonics is the physical science of light based on the concept of "photons" introduced by Albert Einstein in the early $20^{\text {th }}$ century. Einstein introduced this concept in the "particle-wave duality" discussion with Niels Bohr to demonstrate that even light has particle properties (mass and momentum) and wave properties (frequency). That concept became a metaphor and from that time on a beam of light has been generally considered as a beam of particles (photons). Which is a wrong understanding. Light particles do not exist. Photons are nothing else but electromagnetic complex wave configurations and light particles are not like "particles" but separated electromagnetic wave packages, 2-dimensionally confined in the directions perpendicular to the direction of propagation and in a perfect equilibrium with the radiation pressure and the inertia of electromagnetic energy in the forward direction, controlling the speed of light. This new theory will explain how electromagnetic wave packages demonstrate inertia, mass and momentum and which forces keep the wave packages together in a way that they can be measured like particles with their own specific mass and momentum. All we know about light, and in generally about any electromagnetic field configuration, has been based only on two fundamental theories. James Clerk Maxwell introduced in 1865 the "Theory of Electrodynamics" with the publication: "A Dynamical Theory of the Electromagnetic Field" and Albert Einstein introduced in 1905 the "Theory of Special Relativity" with the publication: "On the Electrodynamics of Moving Bodies" and in 1913 the "Theory of General Relativity" with the publication: "Outline of a Generalized Theory of Relativity and of a Theory of Gravitation". However, both theories are not capable to explain the property of electromagnetic mass and in specific the anisotropy of the phenomenon of electromagnetic mass presented e.g. in a LASER beam. To understand what electromagnetic inertia and the corresponding electromagnetic mass is and how the anisotropy of electromagnetic mass can be explained and how it has to be defined, a New Theory about Light has to be developed. A part of this "New Theory about Light", based on Newton's well known law in 3 dimensions will be published in this article in an extension into 4 dimensions. Newton's 4-dimensional law in the 3 spatial dimensions results in an improved version of the classical Maxwell equations and Newton's law in the $4^{\text {th }}$ dimension (time) results in the quantum mechanical Schrödinger wave equation (at non-relativistic velocities) and the relativistic Dirac equation.
\end{abstract}

Keywords: General Relativity, Classical Electrodynamics, Relativistic Quantum Physics, Electromagnetic-Gravitational Interaction, Dirac Equation, Maxwell Tensor, Energy Momentum Tensor

\section{Introduction}

Electromagnetic pulses propagate generally as spherical waves in every direction with the same universal constant, the speed of light. However, photons propagate in a very different way. Photons propagate like particles in one single direction. Thus, the essential question is: "Where does the fundamental boundary between "electromagnetic pulses" and "photons" exist. All we know about light, and in general about any electromagnetic field configuration, has been based only on two fundamental theories. James Clerk Maxwell introduced in 1865 the "Theory of Electrodynamics" with the publication: "A Dynamical Theory of the Electromagnetic Field" and Albert Einstein introduced in 1905 the "Theory of Special Relativity" with the publication: "On the Electrodynamics of Moving Bodies" 
and in 1913 the "Theory of General Relativity" with the publication: "Outline of a Generalized Theory of Relativity and of a Theory of Gravitation". However, both theories are not capable to explain the property of electromagnetic mass and in specific the anisotropy of the phenomenon of electromagnetic mass. To understand what electromagnetic inertia and the corresponding electromagnetic mass is and how the anisotropy of electromagnetic mass can be explained and how it has to be defined, a New Theory about Light has to be developed. A part of this New Theory about Light will be published in this article.

The New Theory about Light has been based on one single fundamental property of our Universe. The unique property that there has always been, is always and will always be a perfect equilibrium within our Universe. Isaac Newton has discovered this fundamental physical law already 300 years ago by his third law in physics. "For every action there is an equal and opposite reaction". In the New Theory this law of Equilibrium has been extended for any arbitrary Electromagnetic Field Configuration, which requires the fundamental Universal Property: "The total algebraic sum of all force densities will always equal zero at any time at any spatial coordinate in any spatial direction".

To develop a set of 4 electromagnetic equations, describing all the force densities within any arbitrary electromagnetic field configuration, the Divergence of the 4Dimensional Stress-Energy Tensor has been taken, resulting in the 4-Dimensional Electromagnetic Vector Equation with the fundamental requirement: "The the total algebraic sum of all force densities equals zero at any time at any spatial coordinate in any spatial direction"

\section{The Unified 4-Dimensional Hyperspace Equilibrium Equation (Newton's Law in 4 Dimensions)}

The 4-dimensional Electromagnetic "Maxwell Tensor" has been defined by:

$$
\mathrm{F}_{a b}=\partial_{b} \varphi_{a}-\partial_{a} \varphi_{b}
$$

The 4-dimensional Electromagnetic "Energy Momentum Tensor" has been defined by:

$$
T^{a b}=\frac{1}{\mu_{0}}\left[F_{a c} F^{c b}+\frac{1}{4} \delta_{a b} F_{c d} F^{c d}\right]
$$

The 4-dimensional divergence of the Energy Momentum Tensor equals the 4-dimensional Force Density 4-vector:

$$
f^{a}=\partial_{b} \mathrm{~T}^{a b}
$$

The new theory has been based on the fundamental concept of Harmony in which all force densities in the Universe have been counter balanced by equal and opposite directed force densities resulting in a net force density equals zero (Newton's $3^{\text {rd }}$ law) and a final set of 4 Electromagnetic Equations, representing the 4-Dimensional equation of Newton (expressed in force densities) applied to an electromagnetic field, results in:

$$
f^{a}=\partial_{b} \mathrm{~T}^{a b}=0
$$

In the absence of any Gravity, the force density $f^{a}$ in the 3 directions of the 3 coordinates of the chosen 3 -coordinate system follows from the (4-dimensional) Divergence of the (4-dimensional) Stress Energy Tensor ${ }^{(8,9,38)}$ (3).

The Divergence of a Vector equals a Scalar. The Divergence of a Tensor equals a Vector. The 4-dimensional Divergence of the 4-dimensional Stress Energy Tensor (4) equals the 4-dimensional Force-density Vector $f^{a}$.

The first 3 terms of the 4-dimensional Force-density vector equal the force densities in the corresponding 3 dimensions of the chosen Coordinate System. The $4^{\text {th }}$ component equals the Electromagnetic Poynting's Theorem (5) in the time direction $\mathrm{x}_{4}$ (Continuity Equation), which is the $4^{\text {th }}$ term of Newton's equation.

To calculate the equilibrium conditions to present the force densities in the Electromagnetic Field Configuration, the first 3 terms of the 4-dimensional Force-density vector are being used. By re-arranging the first 3 terms of the (4-dimensional Divergence) of the (4-dimensional) Stress Energy Tensor (4) results in equation (5) in the spatial directions $\left(\mathrm{x}_{1}, \mathrm{x}_{2}, \mathrm{x}_{3}\right)$ for the 3-dimensional force density $f^{a}$ within the Electromagnetic Field Configuration which is an improved version of Maxwell's 4 equations describing in a classical way the electromagnetic field.

$$
-\frac{1}{c^{2}} \frac{\partial(\overline{\mathrm{E}} \times \overline{\mathrm{H}})}{\partial t}+\varepsilon_{0} \overline{\mathrm{E}}(\nabla . \overline{\mathrm{E}})-\varepsilon_{0} \overline{\mathrm{E}} \times(\nabla \times \overline{\mathrm{E}})+\mu_{0} \overline{\mathrm{H}}(\nabla . \overline{\mathrm{H}})-\mu_{0} \overline{\mathrm{H}} \times(\nabla \times \overline{\mathrm{H}})=\overline{0}
$$

Equation (5) in the 3 spatial directions $\left(\mathrm{x}_{1}, \mathrm{x}_{2}, \mathrm{x}_{3}\right)$ represents the 3-dimensional force density $f^{a}$ in a coordinate-free vector equation in the absence of any Gravity, which is the 3 Dimensional representation of Newton's $3^{\text {rd }}$ law expressed in electromagnetic force densities within an arbitrary electromagnetic field configuration and simultaneously represents the improved version of Maxwell's 4 equations.

Equation (5) is a much more powerful equation than the wellknown 4 Maxwell equations and offers solutions for many physically well-known electromagnetic field configurations like a LASER beam and the projection of a slide on a screen which cannot be mathematically described by Maxwell's equations. Because LASER beams and projected images can never be divergence free for the electric field and/or the magnetic field intensities at any place at any time.

The scope of Newton's $3^{\text {rd }}$ law (the algebraic sum of all the forces equals zero) has been underestimated. Newton's $3^{\text {rd }}$ law expressed in force densities and applied to the electromagnetic force densities within a beam of light results in a much more powerful equation than the well-known 4 
Maxwell equations and also completely includes all the possible solutions of the Maxwell equations.

According Newton's $3^{\text {rd }}$ law, the fundamental requirement of a perfect equilibrium at any place at any time in any direction, the algebraic sum of all the different force densities $\vec{f}$ has to be equal zero for any physical possible electromagnetic field configuration (particles and fields).

$$
\begin{gathered}
\text { 3-Dimensional Space Domain } \\
\left(\begin{array}{c}
\mathrm{x}_{3} \\
\mathrm{x}_{2} \\
\mathrm{x}_{1}
\end{array}\right) \quad-\frac{1}{c^{2}} \frac{\partial(\overline{\mathrm{E}} \times \overline{\mathrm{H}})}{\partial t}+\varepsilon_{0} \overline{\mathrm{E}}(\nabla . \overline{\mathrm{E}})-\varepsilon_{0} \overline{\mathrm{E}} \times(\nabla \times \overline{\mathrm{E}})+ \\
+\mu_{0} \overline{\mathrm{H}}(\nabla \cdot \overline{\mathrm{H}})-\mu_{0} \overline{\mathrm{H}} \times(\nabla \times \overline{\mathrm{H}})=\overline{0}
\end{gathered}
$$

It is fundamental to realize that 3 different kind of separate interactions of the force densities in (5) are being involved within this resulting perfect equilibrium. Magnetic-Magnetic interaction (terms B-4 and B-5), Electric-Electric interaction (terms B-2 and B-3) and Electric-Magnetic interaction (term B-1) which equals the time-derivative of the the Poynting vector and represents the inertia of electromagnetic radiation and is comparable with Newton's second law of motion $(\mathrm{F}=$ $\mathrm{m} \mathrm{a}=\mathrm{m} \mathrm{d} \mathrm{p} \mathrm{/} \mathrm{dt)} \mathrm{for} \mathrm{mechanical} \mathrm{objects.}$
The force density vector $f^{a}$ is 4-dimensional. The 4dimensional Divergence of the 4-dimensional Stress Energy Tensor (4) equals the 4-dimensional Force-density Vector $f^{a}$ and can be written as the Electromagnetic "4Dimensional Hyperspace Equilibrium Equation" (Newton's $3^{\text {rd }}$ Law in 4 Dimensions expressed in electromagnetic force densities):

$$
\begin{aligned}
& \text { Energy-Time Domain } \\
& \left(\mathrm{x}_{4}\right) \quad \nabla \cdot(\overline{\mathrm{E}} \times \overline{\mathrm{H}})+\frac{1}{2} \frac{\partial\left(\varepsilon_{0}(\overline{\mathrm{E}} \cdot \overline{\mathrm{E}})+\mu_{0}(\overline{\mathrm{H}} \cdot \overline{\mathrm{H}})\right)}{\partial t}=0 \\
& \text { 3-Dimensional Space Domain } \\
& \text { B-1 B-2 B-3 } \\
& \left(\begin{array}{c}
\mathrm{x}_{3} \\
\mathrm{x}_{2} \\
\mathrm{x}_{1}
\end{array}\right) \quad \begin{array}{c}
-\frac{1}{c^{2}} \frac{\partial(\overline{\mathrm{E}} \times \overline{\mathrm{H}})}{\partial t}+\varepsilon_{0} \overline{\mathrm{E}}(\nabla . \overline{\mathrm{E}})-\varepsilon_{0} \overline{\mathrm{E}} \times(\nabla \times \overline{\mathrm{E}})+ \\
+\mu_{0} \overline{\mathrm{H}}(\nabla \cdot \overline{\mathrm{H}})-\mu_{0} \overline{\mathrm{H}} \times(\nabla \times \overline{\mathrm{H}})=\overline{0}
\end{array}
\end{aligned}
$$

An electromagnetic field which is in a perfect equilibrium with itself and its surrounding at any space and time in any direction, fulfills the necessary requirements for the physical possibility of the existence of this field. Under that condition Equation (4) transforms into the "Unified 4-Dimensional Hyperspace Equilibrium Equation" (7) which is the 4Dimensional representation of Newton's $3^{\text {rd }}$ Law expressed in electromagnetic force densities.

To extend Field Equation (5) into an equilibrium within a multi-dimensional curved Space-Time continuum unifying different fields like gravity and electromagnetism, the transformation has been realized by the transformation of the resulting force-densities within the 4-Dimensional SpaceTime continuum. The Unification of the Electromagnetic Fields with the Gravitational fields results in the "Relativistic Gravitational Electromagnetic Equilibrium Equation" (RGEE) within a gravitational field $\vec{g}$ in the 3-dimensional (spatial) representation:

$$
\begin{gathered}
-\frac{1}{c^{2}} \frac{\partial(\bar{E} \times \bar{H})}{\partial t}+\varepsilon_{0} \bar{E}(\nabla . \bar{E})-\varepsilon_{0} \bar{E} \times(\nabla \times \bar{E})+\mu_{0} \bar{H}(\nabla \cdot \bar{H})- \\
-\mu_{0} \bar{H} \times(\nabla \times \bar{H})-\frac{1}{2} \varepsilon_{0}{ }^{2} \mu_{0}(\bar{E} \cdot \bar{E}) \overline{\mathrm{g}}-\frac{1}{2} \varepsilon_{0} \mu_{0}{ }^{2}(\bar{H} \cdot \bar{H}) \overline{\mathrm{g}}=\overline{0}
\end{gathered}
$$

It is fundamental to realize that Equation (8) is only a part of the 4-Dimensional Time-Space Continuum Equation related to the spatial coordinates.

The 4-Dimensional Divergence of the 4-Dimensional Stress-Energy Tensor (2) results in the 4-Dimensional Force Density $f^{a}$ Vector Equation (3). Fulfilling the fundamental law of Universal Equilibrium (Newton's $3^{\text {rd }}$ law) which can be expressed as: "The total algebraic sum of all force densities will always equal zero at any time at any spatial coordinate in any spatial direction" results in the fundamental 4-Dimensional vector equation (4). The first 3 terms in the 3Dimensional Space Domain of the 4-Dimensional vector Equation (7) have been presented in (5) and (6) and represent the improved Maxwell equations.

The $4^{\text {th }}$ term in the Energy-Time domain, described in equation (7), presents the Continuum Equation (Ref. [1, 
2]). By introducing the complex field notations for the electric field and the magnetic field in Equation (7), the $4^{\text {th }}$ term transforms (Ref. [3-6]) into the well-known relativistic quantum mechanical Dirac Equation (ref. [79]) and at low velocities into the quantum mechanical Schrödinger Wave Equation. (Ref. [10], Equation (56) page 208).

The Poynting Theorem ( $4^{\text {th }}$ term in equation (7)) can be rewritten by introducing the vector functions $\bar{\phi}$ and the complex conjugated vector function $\bar{\phi}^{*}$ in which:

$$
\bar{\phi}=\frac{1}{\sqrt{2 \mu}}\left(\overline{\mathrm{B}}+\mathrm{i} \frac{\overline{\mathrm{E}}}{\mathrm{c}}\right)
$$

$\bar{B}$ equals the magnetic induction, $\bar{E}$ the electric field intensity and $\mathrm{c}$ the speed of light. The complex conjugated vector function equals:

$$
\overline{\phi^{*}}=\frac{1}{\sqrt{2 \mu}}\left(\overline{\mathrm{B}}-\mathrm{i} \frac{\overline{\mathrm{E}}}{c}\right)
$$

The dot product equals the electromagnetic energy density w:

$$
\bar{\phi} \cdot \overline{\phi^{*}}=\frac{1}{2 \mu}\left(\overline{\mathrm{B}}+\mathrm{i} \frac{\overline{\mathrm{E}}}{c}\right) \cdot\left(\overline{\mathrm{B}}-\mathrm{i} \frac{\overline{\mathrm{E}}}{c}\right)=\frac{1}{2} \mu \mathrm{H}^{2}+\frac{1}{2} \varepsilon \mathrm{E}^{2}=\mathrm{w}
$$

The cross product is proportional to the Poynting vector (ref. [11], page 202, equation (15).

$$
\bar{\phi} \times \overline{\phi^{*}}=\frac{1}{2 \mu}\left(\overline{\mathrm{B}}+\mathrm{i} \frac{\overline{\mathrm{E}}}{c}\right) \times\left(\overline{\mathrm{B}}-\mathrm{i} \frac{\overline{\mathrm{E}}}{c}\right)=\mathrm{i} \sqrt{\varepsilon \mu} \overline{\mathrm{E}} \times \overline{\mathrm{H}}=\mathrm{i} \sqrt{\varepsilon \mu} \overline{\mathrm{S}}
$$

Substituting (11) and (12) in the $4^{\text {th }}$ term (Energy-Time domain) in equation (7) results in the 4-Dimensional Hyperspace Equilibrium Equation (Newton's Law in 4 Dimensions) (13):

$$
\begin{gathered}
\text { Energy-Time Domain } \\
\left.\left(\mathrm{x}_{4}\right) \quad-\frac{i}{\sqrt{\varepsilon_{0} \mu_{0}}} \nabla \cdot \bar{\phi} \times \bar{\phi}\right)=-\frac{\partial \bar{\phi} \cdot \bar{\phi}^{*}}{\partial t} \\
\text { 3-Dimensional Space Domain } \\
\left(\begin{array}{c}
\mathrm{x}_{3} \\
\mathrm{x}_{2} \\
\mathrm{x}_{1}
\end{array}\right) \quad-\frac{1}{c^{2}} \quad \frac{\partial(\overline{\mathrm{E}} \times \overline{\mathrm{H}})}{\partial t}+\varepsilon_{0} \overline{\mathrm{E}}(\nabla \cdot \overline{\mathrm{E}})-\varepsilon_{0} \overline{\mathrm{E}} \times(\nabla \times \overline{\mathrm{E}})+ \\
+\mu_{0} \overline{\mathrm{H}}(\nabla \cdot \overline{\mathrm{H}})-\mu_{0} \overline{\mathrm{H}} \times(\nabla \times \overline{\mathrm{H}})=\overline{0}
\end{gathered}
$$

To transform the electromagnetic vector wave function $\bar{\phi}$ into a scalar (spinor or one-dimensional matrix representation), the Pauli spin matrices $\sigma$ and the following matrices (Ref. [10] page 213, equation (99) have been introduced:

$$
\bar{\alpha}=\left[\begin{array}{ll}
0 & \sigma \\
\sigma & 0
\end{array}\right] \quad \text { and } \quad \bar{\beta}=\left[\begin{array}{cc}
\delta_{a b} & 0 \\
0 & -\delta_{a b}
\end{array}\right]
$$

Then equation (13) can be rewritten as the 4-Dimensional Hyperspace Equilibrium Dirac Equation (Newton's $3^{\text {rd }}$ Law in 4 Dimensions in a quantum mechanical presentation) (15):

$$
\begin{aligned}
& \text { Energy-Time Domain } \\
& \left(\mathrm{x}_{4}\right) \quad\left(\frac{\mathrm{imc}}{h} \bar{\beta}+\bar{\alpha} \cdot \nabla\right) \psi=-\frac{1}{c} \frac{\partial \psi}{\partial t} \\
& \text { 3-Dimensional Space Domain }
\end{aligned}
$$

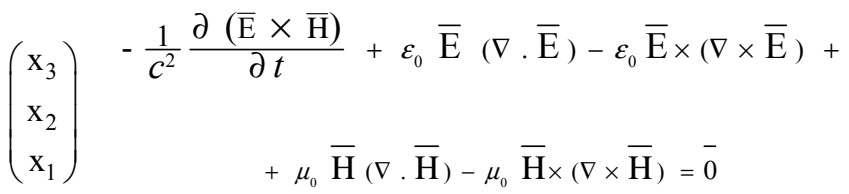

The $4^{\text {th }}$ term in the Energy-Time domain, described in equation (7) represents the relativistic quantum mechanical Dirac equation (15) (which equals equation (102) page 213 in

\section{Ref. [10]).}

The 4 Equations together (3 Equations for the separate space coordinates) and the Dirac/Schrödinger Equation (for 
the time-coordinate) describe the Unification in a perfect Equilibrium of the different Fields (Electromagnetic Field, Gravitational Field)

\section{Fundamental Solutions of the Unified 4-Dimensional Hyperspace Equilibrium Equation}

The required Electromagnetic Field Configuration for a perfect Equilibrium in Space and Time for the total algebraic sum of all the force densities being involved (electromagnetic radiation pressure, electromagnetic inertia and electromagnetic interaction) follows from the "Unified 4Dimensional Hyperspace Equilibrium Equation" (5).

\subsection{The Propagation of a Beam of Light in One Single Direction with an Arbitrary Electromagnetic Intensity Division}

The first example for the many possible Electromagnetic Field Configuration as a solution of the "Unified 4Dimensional Hyperspace Equilibrium Equation" represents a beam of light propagating in the z-direction and an arbitrary division $\mathrm{f}(\mathrm{x}, \mathrm{y})$ of intensity in the $(\mathrm{x}, \mathrm{y})$ plane. The solution for the Electric Field Intensity in Cartesian Coordinates $\{t, x, y, z\}$ for the Electric Field Component e $(x, y, z, t)$ equals:

$$
\left(\begin{array}{l}
e_{x} \\
e_{y} \\
e_{z}
\end{array}\right)=\left(\begin{array}{c}
f(x, y) g\left(t-\left(\frac{\mathrm{K}_{1}}{z}+1\right) z \sqrt{\varepsilon_{0} \mu_{0}}\right) \\
0 \\
0
\end{array}\right)
$$

The required Electromagnetic Field Configuration for a perfect Equilibrium in Space and Time follows from the dynamic equilibrium equation (5) and equals in Cartesian Coordinates $\{x, y, z, t\}$ for the Magnetic Field Components $\mathrm{m}$ $(t, x, y, z)$ :

$$
\left(\begin{array}{l}
m_{x} \\
m_{y} \\
m_{z}
\end{array}\right)=\sqrt{\frac{\varepsilon_{0}}{\mu_{0}}}\left(f(x, y) g\left(t-\left(\frac{\mathrm{K}_{1}}{z}+1\right) z \sqrt{\varepsilon_{0} \mu_{0}}\right)\right)
$$

In which $K_{1}$ is an arbitrary constant. For the divergencefree function $f(x, y)=1$, the solutions (16) and (17) are also the solutions for the well-known Maxwell Equations. For the non-divergence-free functions $f(x, y)$, the solutions (16) and (17) are not solutions for the Maxwell Equations, which requires divergence-free electromagnetic waves, propagating with the speed of light $c=1 / \sqrt{\varepsilon_{0} \mu_{0}}$, in the absence of any matter. But they are solutions of the Dynamic Equilibrium Equation (5) and clearly do exist in physics. Comparable with the projection of a slide with a beamer on a flat screen in the $\mathrm{z}$-direction. In which the slide has an arbitrary intensity-division $f(x, y)$. The information $f(x, y)$ on the slide propagates with the speed of light $c=1 / \sqrt{\varepsilon_{0} \mu_{0}}$ towards the screen in the z-direction in this example while the speed of propagation in the $\mathrm{x}$ - and $\mathrm{y}$ direction equals zero.

\subsection{The Preeminence of the Unified 4-Dimensional Hyperspace Equilibrium Equation Compared to the Classical 4 Maxwell Equations}

James Clerk Maxwell (1831-1879) was one of the greatest scientists who have ever lived. To him we owe the most significant discovery of our age - the theory of electromagnetism in which theory the electric field and the magnetic field has been unified as one physical phenomenon. $\mathrm{He}$ is rightly acclaimed as the father of modern physics. $\mathrm{He}$ also made fundamental contributions to mathematics, astronomy and engineering.

Albert Einstein said: "The special theory of relativity owes its origins to Maxwell's equations of the electromagnetic field." Einstein also said: "Since Maxwell's time, physical reality has been thought of as represented by continuous fields, and not capable of any mechanical interpretation (Ref. [12-15]). This change in the conception of reality is the most profound and the most fruitful that physics has experienced since the time of Newton" Einstein called James Clerk Maxwell the greatest physicists of his century.

And indeed, James Clerk Maxwell fully deserves all the credits that has been given to him. But we should not forget Isaac Newton. Because Isaac Newton has discovered the most fundamental law of our universe. The fundamental law of Equilibrium. And on this classical fundamental law of Equilibrium, the "New Theory" has been grounded. And based on that single law of Equilibrium the "New Theory" is able to offer mathematical solutions where Maxwell's 4 equations do fail.

The preeminence of the "Unified 4-Dimensional Hyperspace Equilibrium Equation" compared to the 4 Maxwell equations can be easily demonstrated with the simple example of a LASER beam propagating through space. The intensity division for a LASER beam is not Divergence-Free and for that reason not a solution of the 4 Maxwell Equations. However, the basic example of a LASER beam propagating through space is an exact solution of the "Unified 4-Dimensional Hyperspace Equilibrium Equation" which offers solutions in Non- Divergence-Free Electromagnetic Field Configurations.

In general a LASER beam can be described with a Gaussian intensity division for the electromagnetic field in the plane perpendicular to the direction of propagation. 


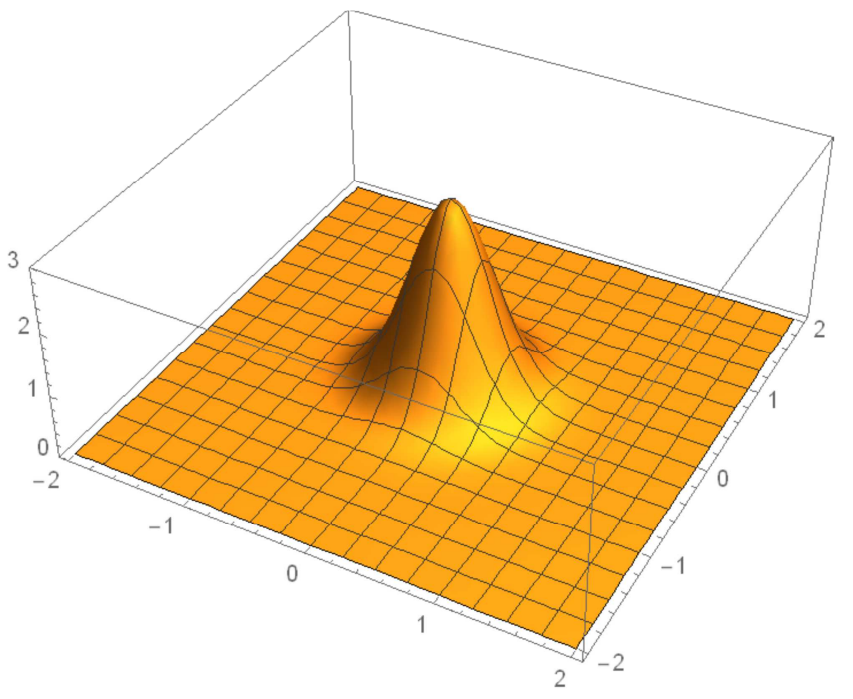

Figure 1. Electromagnetic Field Intensity with a Gaussian division.

The required Electromagnetic Field Configuration (Ref. $[16,17])$, which is a mathematical solution of the "Unified 4Dimensional Hyperspace Equilibrium Equation" (5) equals in Cartesian Coordinates $\{x, y, z, t\}$ for the Electric Field Intensity components $\mathrm{e}(t, x, y, z)$ :

$$
\left(\begin{array}{c}
e_{x} \\
e_{y} \\
e_{z}
\end{array}\right)=\left(\begin{array}{c}
K_{1} \mathrm{e}^{-K_{2}\left(x^{2}+y^{2}\right)} \\
\operatorname{Sin}\left(\omega\left(t-z \sqrt{\varepsilon_{0} \mu_{0}}\right)\right) \\
0 \\
0
\end{array}\right)
$$

The required Electromagnetic Field Configuration (Ref. $[16,17])$ for a perfect Equilibrium in Space and Time follows from the dynamic equilibrium equation (5) and equals in Cartesian Coordinates $\{x, y, z, t\}$ for the Magnetic Field Intensity components $\mathrm{m}(t, x, y, z)$ :

$$
\left(\begin{array}{l}
m_{x} \\
m_{y} \\
m_{z}
\end{array}\right)=\sqrt{\frac{\varepsilon_{0}}{\mu_{0}}}\left(\begin{array}{c}
0 \\
K_{1} \mathrm{e}^{-K_{2}\left(x^{2}+y^{2}\right)} \operatorname{Sin}\left(\omega\left(t-z \sqrt{\varepsilon_{0} \mu_{0}}\right)\right) \\
0
\end{array}\right)
$$

In which $K_{1}$ is an arbitrary constant. The Gaussian division cannot be a solution of the Maxwell Equations, because the divergence of the Electric field intensity does not equal zero.

$$
\nabla \cdot \overline{\mathrm{E}}=-2 \mathrm{e}^{-\mathrm{K}_{2}\left(\mathrm{x}^{2}+\mathrm{y}^{2}\right)} \times \mathrm{K}_{1} \mathrm{~K}_{2} \operatorname{Sin}\left(\left(t-z \sqrt{\varepsilon_{0} \mu_{0}}\right) \omega\right)
$$

And the Divergence of the Magnetic field intensity does not equal zero.

$$
\nabla . \overline{\mathrm{H}}=-\sqrt{\frac{\varepsilon_{0}}{\mu_{0}}}\left(2 \mathrm{e}^{-\mathrm{K}_{2}\left(\mathrm{x}^{2}+\mathrm{y}^{2}\right)} \mathrm{y} \mathrm{K}_{1} \mathrm{~K}_{2} \operatorname{Sin}\left(\left(t-z \sqrt{\varepsilon_{0} \mu_{0}}\right) \omega\right)\right)
$$

$$
\begin{array}{ccr}
\text { For } \quad \text { the } \quad \text { non-divergence-free } & \text { functions } \\
f(x, y)=K_{1} \mathrm{e}^{-K_{2}\left(x^{2}+y^{2}\right)} \operatorname{Sin}\left(\omega\left(t-z \sqrt{\varepsilon_{0} \mu_{0}}\right)\right), \text { the }
\end{array}
$$

solutions (18) and (19) are not solutions for the Maxwell Equations, which requires divergence-free electromagnetic waves, propagating with the speed of light $c=1 / \sqrt{\varepsilon_{0} \mu_{0}}$, in the absence of any matter. But they are solutions of the Dynamic Equilibrium Equation (5) and clearly do exist in physics. Since 1960 the LASER beam is a physical reality when the first LASER has been built by Theodore $\mathrm{H}$. Maiman at the Hughes Research Laboratories. A physical phenomenon that could not be explained by Maxwell's equations but is an existing solution of the Dynamic Equilibrium Equation (5).

\section{Electromagnetic Mass (Inertia)}

In a classical way mass (inertia) has been defined by Newton's $2^{\text {nd }}$ law. Einstein already discovered that mass (inertia, $\mathrm{m}$ ) and energy $(\mathrm{W})$ are related by $\mathrm{W}=\mathrm{m} \mathrm{c}^{2}$. In this chapter a new definition of mass will be represented which is based on the relativistic changes in the radiation pressure of confined electromagnetic radiation during acceleration or deceleration.

\subsection{Confined Electromagnetic Mass (Inertia)}

The definition of mass is related to concept of inertia, defined by Newton's second law. For this reason, free electromagnetic radiation cannot be related to the concept of mass because it is impossible to accelerate or to decelerate free electromagnetic radiation. The speed of light is a universal constant. Only confined electromagnetic radiation can be accelerated or decelerated and for this reason electromagnetic mass (inertia) can only be assigned to confined electromagnetic radiation.

\subsubsection{The Origin of Electromagnetic Mass (Inertia)}

Newton described in his second law of motion the classical definition of mass (inertia). However, it is impossible to calculate with Newton's second law of motion the (electromagnetic) mass of a beam of light. Because the speed of light is a universal constant which follows from Albert Einstein's Theory of Special Relativity, it is impossible to accelerate or to slow a beam of light and for that reason it is impossible to determine the electromagnetic mass of a beam of light (free electromagnetic radiation) by Newton's second law.

To calculate the electromagnetic mass of free or confined electromagnetic radiation, the fundamental concept of the 
New Theory has been used that the Universe is in a perfect Equilibrium and that any electromagnetic field configuration is in a perfect equilibrium with itself and its surrounding. From this fundamental concept follows a different definition of (confined) electromagnetic mass.

Electromagnetic mass (or inertia) has been determined by the relativistic Lorentz transformation of the radiation pressures in all different directions and the disturbance of a uniform motion (or position at rest) of confined electromagnetic radiation results in a relativistic effect which we measure (experience) as electromagnetic mass (inertia).

The mass in [kg] of an object will be generally measured by acceleration (or deceleration) of the object according Newton's second law of motion. In the theory of special relativity, the speed of light is a fundamental constant and the intensity of the light is not a universal constant.

To calculate the relativistic mass of Confined Electromagnetic Radiation, we start with a thought experiment in which a beam of light is propagating between two $100 \%$ reflecting mirrors, indicated as Mirror $\mathrm{A}$ and Mirror B. Both mirrors are part of a rigid construction and the relative velocity between both mirrors always equals zero. The results of this calculation will be be generalized for any kind of electromagnetic radiation which has been confined by its own electromagnetic and gravitational field. When the speed of the observer has the same speed as the speed of the light source, then the observer and the light source are relative at rest. And the same light intensity will be measured at the location of the emitter and at the location of the observer.

When the observer moves towards the emitter, the intensity of the light at the location of the observer will increase with $\gamma(1+\mathrm{v} / \mathrm{c})$ according the Lorentz transformation in which " $v$ " is the relative velocity between emitter and observer. At low velocities the term $\gamma$ will equal 1 .

When the observer moves away from the emitter, the intensity of the light at the location of the observer will decrease with $\gamma(1-\mathrm{v} / \mathrm{c})$ according the Lorentz transformation. At low velocities the term $\gamma$ will equal 1 .

When light is confined between two $100 \%$ reflecting mirrors, then we can conclude that the speed of both mirrors will always be equal, relative to each other. And at uniform speed, the radiation pressures on both mirrors will be equal and both opposite directed radiation pressures will neutralize.

During acceleration, it will take time for the light to travel with the speed of light between both mirrors. When we consider one mirror as the emitter and the opposite mirror as the observer, we can conclude that the speed of the emitter will be different (when the beam of light leaves the emitter) than the speed of the observer (when the beam of light reaches the observer) because of the time interval, needed for the beam of light to propagate from emitter to observer during the acceleration.

During the acceleration, both opposite oriented radiation pressures on both mirrors will not be equal anymore and they will not neutralize each other anymore. During acceleration, there will be a resulting force according Newton's second law of motion caused by both different radiation pressures.

For the first step in this calculation an imaginary experiment has been used. Two 100\% reflecting mirrors B and $\mathrm{A}$ (both in the $\mathrm{x}-\mathrm{y}$ plane and without any mass) are placed opposite each other at a distance $\Delta \mathrm{x}$ (Ref [10], page 206, figure 1). A single harmonic electromagnetic wave has been confined between both mirrors. Between both mirrors a "Standing Electromagnetic Wave" appears which has been formed by two waves travelling in opposite directions along the z-axis.

The Poynting vector corresponding with the electromagnetic wave propagating along the z-axis in the + direction (positive direction of the z-axis) has been indicated as $\overline{\mathrm{S}}^{+}=\overline{\mathrm{E}}^{+} \times \overline{\mathrm{H}}^{+}$and the Poynting vector corresponding with the electromagnetic wave propagating along the z-axis in the - direction (opposite direction) has been indicated as $\bar{S}^{-}=\overline{\mathrm{E}}^{-} \times \overline{\mathrm{H}}^{-}$.

The system is at rest. The radiation pressures, caused by the confined electromagnetic radiation, on both mirrors A and $\mathrm{B}$ are opposite and equal in magnitude:

$$
\mathrm{P}_{\mathrm{A}}=\frac{2 \mathrm{~S}_{\mathrm{A}}}{\mathrm{c}}=\frac{2 \mathrm{~S}_{\mathrm{B}}}{\mathrm{c}}=\mathrm{P}_{\mathrm{B}}
$$

Einstein has formulated this very well. "Velocities are always relative". To calculate the radiation pressure on Mirror A, the velocities, only relative to Mirror A for the waves with the respective Poynting vectors $\bar{S}^{+}=\overline{\mathrm{E}}^{+} \times \overline{\mathrm{H}}^{+}$ and $\bar{S}^{-}=\overline{\mathrm{E}}^{-} \times \overline{\mathrm{H}}^{-}$, have to be calculated.

The radiation pressure on Mirror A, when Mirror A moves with a velocity $\mathrm{v}$ in the direction of the positive $\mathrm{z}$-axis:

When the system of "Two Mirrors B - A" moves in the direction of the positive $\mathrm{z}$-axis, Mirror A moves in the direction of the positive $\mathrm{z}$-axis and the Poynting vector $\bar{S}^{+}=\overline{\mathrm{E}}^{+} \times \overline{\mathrm{H}}^{+}$will decrease according the Lorentz transformation (Ref. [11], page 56 equation (27)).

$$
\bar{S}_{\mathrm{v}}^{+}=\overline{\mathrm{E}}_{\mathrm{v}}^{+} ? \overline{\mathrm{H}}_{\mathrm{v}}^{+}=\gamma^{2}\left(1-\frac{\mathrm{v}}{\mathrm{c}}\right)^{2}\left(\overline{\mathrm{E}}^{+} ? \overline{\mathrm{H}}^{+}\right)
$$

When the system of "Two Mirrors B - A" moves in the direction of the positive $\mathrm{z}$-axis, Mirror A moves in the direction of the positive $z$-axis the Poynting vector $\bar{S}^{-}=\overline{\mathrm{E}}^{-} \times \overline{\mathrm{H}}^{-}$will increase according the Lorentz transformation (ref. [11], page 56 equation (28)).

$$
\bar{S}_{\mathrm{v}}^{-}=\overline{\mathrm{E}}_{\mathrm{v}}^{-} ? \overline{\mathrm{H}}_{\mathrm{v}}^{-}=\gamma^{2}\left(1+\frac{\mathrm{v}}{\mathrm{c}}\right)^{2}\left(\overline{\mathrm{E}}^{+} ? \overline{\mathrm{H}}^{+}\right)
$$

The total radiation pressure, caused by the confined electromagnetic radiation, on mirror A equals: 


$$
\mathrm{P}_{\mathrm{A}}=\frac{\mathrm{S}_{A}^{+}+\mathrm{S}_{A}^{-}}{\mathrm{c}}=\frac{\gamma^{2}\left(\left(1-\frac{\mathrm{v}}{\mathrm{c}}\right)^{2}+\left(1+\frac{\mathrm{v}}{\mathrm{c}}\right)^{2}\right)\left(\overline{\mathrm{E}}^{+} ? \overline{\mathrm{H}}^{+}\right)}{\mathrm{c}}
$$

\subsubsection{The Radiation Pressure on Mirror B When Mirror B Moves with a Velocity $v$ in the Direction of the \\ Positive Z-axis}

When the system of "Two Mirrors B - A" moves in the direction of the positive $\mathrm{z}$-axis, Mirror $\mathrm{B}$ moves in the direction of the positive $z$-axis and the Poynting vector $\bar{S}^{-}=\overline{\mathrm{E}}^{-} \times \overline{\mathrm{H}}^{-}$will increase according the Lorentz transformation (Ref. [11], page 57 equation (30)).

$$
{\overline{S_{\mathrm{v}}}}_{-}^{-}=\overline{\mathrm{E}}_{\mathrm{v}}^{-} ? \overline{\mathrm{H}}_{\mathrm{v}}^{-}=\gamma^{2}\left(1+\frac{\mathrm{v}}{\mathrm{c}}\right)^{2}\left(\overline{\mathrm{E}}^{+} ? \overline{\mathrm{H}}^{+}\right)
$$

When the system of "Two Mirrors B - A" moves in the direction of the positive z-axis, Mirror A moves in the direction of the positive $\mathrm{z}$-axis the Poynting vector $\overline{\mathrm{S}}^{+}=\overline{\mathrm{E}}^{+} \times \overline{\mathrm{H}}^{+}$will increase according the Lorentz transformation (Ref. [11], page 57 equation (31)).

$$
\bar{S}_{\mathrm{v}}^{+}=\overline{\mathrm{E}}_{\mathrm{v}}^{+} ? \overline{\mathrm{H}}_{\mathrm{v}}^{+}=\gamma^{2}\left(1-\frac{\mathrm{v}}{\mathrm{c}}\right)^{2}\left(\overline{\mathrm{E}}^{+} ? \overline{\mathrm{H}}^{+}\right)
$$

The total radiation pressure, caused by the confined electromagnetic radiation, on mirror B equals (Ref. [11], page 57 equation (32)).:

$$
\mathrm{P}_{\mathrm{A}}=\frac{\mathrm{S}_{A}^{+}+\mathrm{S}_{A}^{-}}{\mathrm{c}}=\frac{\gamma^{2}\left(\left(1+\frac{\mathrm{v}}{\mathrm{c}}\right)^{2}+\left(1-\frac{\mathrm{v}}{\mathrm{c}}\right)^{2}\right)\left(\overline{\mathrm{E}}^{+} ? \overline{\mathrm{H}}^{+}\right)}{\mathrm{c}}
$$

$\mathrm{P}_{\mathrm{A}}$ and $\mathrm{P}_{B}$ are still equal in magnitude and both in opposite direction and still cancel each other. The system fulfils Newton's first law of motion.

\subsubsection{Newton's Second Law of Motion (Inertia) for Confined Electromagnetic Radiation}

When the system of "Two Mirrors B - A" accelerates, the velocity increases with $\Delta \mathrm{v}$ in a time interval $\Delta \mathrm{t}$. At time $\mathrm{t}$ the radiation pressures on mirror $\mathrm{A}$ and mirror $\mathrm{B}$ are presented in two studies $[10,11]$. At time $t+\Delta t$ the radiation pressures on Mirror A and Mirror B will different:

The radiation pressure at time $\mathrm{t}+\Delta \mathrm{t}$ caused by the confined electromagnetic radiation, on mirror A equals (Ref. [11], page 59 equation (33)):

$$
\mathrm{P}_{\mathrm{A}}=\frac{\mathrm{S}_{A}^{+}+\mathrm{S}_{A}^{-}}{\mathrm{c}}=\frac{\gamma^{2}\left(\left(1+\frac{(\mathrm{v})}{\mathrm{c}}\right)^{2}+\left(1-\frac{(\mathrm{v}+\Delta \mathrm{v})}{\mathrm{c}}\right)^{2}\right)\left(\overline{\mathrm{E}}^{+} ? \overline{\mathrm{H}}^{+}\right)}{\mathrm{c}}
$$

Because the wave with Poynting vector $\overline{\mathrm{S}}^{+}=\overline{\mathrm{E}}^{+} \times \overline{\mathrm{H}}^{+}$ has left Mirror B at " $\mathrm{t}$ " and during the time interval $\Delta \mathrm{t}$ the magnitude of $\overline{\mathrm{E}}_{\mathrm{t}}^{+}=\left(1+\frac{\mathrm{v}}{\mathrm{c}}\right) \overline{\mathrm{E}}^{+}$and $\overline{\mathrm{H}}_{\mathrm{t}}^{+}=\left(1+\frac{\mathrm{v}}{\mathrm{c}}\right) \overline{\mathrm{H}}^{+}$

$$
\mathrm{P}_{B}=\frac{\mathrm{S}_{B}^{+}+\mathrm{S}_{B}^{-}}{\mathrm{c}}=\frac{\gamma^{2}\left(\left(1+\frac{(\mathrm{v}+\Delta \mathrm{v})}{\mathrm{c}}\right)^{2}+\left(1-\frac{(\mathrm{v})}{\mathrm{c}}\right)^{2}\right)\left(\overline{\mathrm{E}}^{+} ? \overline{\mathrm{H}}^{+}\right)}{\mathrm{c}}
$$

Because the wave with Poynting vector $\bar{S}^{-}=\overline{\mathrm{E}}^{-} \times \overline{\mathrm{H}}^{-}$ has left Mirror A at " $\mathrm{t}$ " and during the time interval $\Delta \mathrm{t}$ the magnitude of

$$
\overline{\mathrm{E}}_{\mathrm{t}}^{-}=\left(1+\frac{\mathrm{v}}{\mathrm{c}}\right) \overline{\mathrm{E}}^{-} \text {and } \overline{\mathrm{H}}_{\mathrm{t}}^{-}=\left(1+\frac{\mathrm{v}}{\mathrm{c}}\right) \overline{\mathrm{H}}^{-}
$$

has not changed. has not changed.

The radiation pressure at time $\mathrm{t}+\Delta \mathrm{t}$ caused by the confined electromagnetic radiation, on mirror B equals (Ref. [11], page 59 equation (34)):
The radiation pressures on Mirror A and Mirror B do not counterbalance each other anymore and the resulting radiation pressure equals (Ref. [11], page 60 equation (35)):

$$
\mathrm{P}_{\mathrm{B}}-\mathrm{P}_{\mathrm{A}}=\frac{\gamma^{2}(4 \Delta \mathrm{v}) \mathrm{S}}{\mathrm{c}^{2}}
$$

Equation (21) can be written as (Ref. [11], page 60 equation (36)) 


$$
\mathrm{P}_{\mathrm{B}}-\mathrm{P}_{\mathrm{A}}=\frac{\gamma^{2}(4 \Delta \mathrm{v}) \mathrm{S}}{\mathrm{c}^{2}}=\frac{\gamma^{2}\left(4 \frac{\Delta \mathrm{v}}{\Delta \mathrm{t}}\right) \mathrm{S} \Delta \mathrm{t}}{\mathrm{c}^{2}}=\gamma^{2} \frac{\mathrm{W}}{\mathrm{c}^{2}} \mathrm{a}=\gamma^{2} \mathrm{ma}
$$

In which the acceleration $\mathrm{a}=\frac{\Delta \mathrm{v}}{\Delta \mathrm{t}}$ and the inertia $\mathrm{m}=\frac{\mathrm{W}}{\mathrm{c}^{2}}$. At non-relativistic velocities $\gamma=1$ and (32) turns into the Newton's second law of motion. From (32) also Einstein's famous equation $\mathrm{W}=\mathrm{m} \mathrm{c}^{2}$ follows. In (32) $\mathrm{W}$ is the total confined electromagnetic mass.

Now we can consider electromagnetic confinements without the use of mirrors but electromagnetic confinements through electromagnetic gravitational interaction. By superposition and integration over arbitrary surfaces it is possible to prove that all confined electromagnetic radiation equals (32) and represents electromagnetic mass and follows Newton's second law of motion $(\mathrm{F}=\mathrm{m}$ a).

\subsection{Longitudinal Mass. The Real Light Intensity of the \\ Sun, Measured in Our Solar: System, Including Electromagnetic Gravitational Conversion (EMGC)}

When a beam of light leaves the surface of the sun, the light will travel in the radial direction of the radial gravitational field caused by the sun. This experiment is comparable with throwing up a ball in the direction of the gravitational field on earth. During the rising in height, the kinetic energy of the ball will be transformed into potential energy. The speed of the ball slows down, becomes zero, and during the fall the kinetic potential energy will be transformed again into kinetic energy and the speed of the ball increase again.

Something comparable happens with a beam of light leaving the sun in the direction of the (radial) gravitational field of the sun. But because the speed of light is a universal constant, not the speed of light will be transferred into potential energy, but the electromagnetic energy of the beam of light will be transformed into potential energy. During the time the beam of light leaves the sun, the intensity of the beam of light will become less and the potential energy will increase with the same amount. When a beam of light propagates towards the sun, the electromagnetic field intensity will increase while the potential energy decreases with the same amount.

The required Electromagnetic Field Configuration for a perfect Equilibrium in Space and Time for a Radial Gravitational Field (The Light propagates in the same radialdirection as the radial-direction of the Gravitational Field) follows from the Gravitational Dynamic Equilibrium Equation (8) and equals in Spherical Coordinates $\{r, \theta, \varphi, t\}$ for a gravitational field "g(r)" for the Electric Field Components e $(r, \theta, \varphi, t)$ :

$$
\left(\begin{array}{l}
e_{r} \\
e_{\theta} \\
e_{\varphi}
\end{array}\right)=\left(\begin{array}{cc}
\frac{1}{r} \mathrm{e}^{-1+\frac{\mathrm{G} \mathrm{m}_{1} \varepsilon_{0} \mu_{0}}{2 \mathrm{r}}} & f[\theta, \varphi] \mathrm{h}[t-r \sqrt{\epsilon 0} \sqrt{\mu 0}] \\
0 & 0
\end{array}\right)
$$

In which $\mathrm{G}$ represents the gravitational field intensity [m $\left./ \mathrm{s}^{2}\right]$ of the sun. The required Electromagnetic Field Configuration for a perfect Equilibrium in Space and Time for a Radial Gravitational Field (The Light propagates in the same radial-direction as the radial-direction of the

Gravitational Field) follows from the Gravitational Dynamic Equilibrium Equation (8) and equals in Spherical Coordinates $\{r, \theta, \varphi, t\}$ for a gravitational field " $\mathrm{g}(\mathrm{r})$ " for the Magnetic Field Components $\mathrm{m}(r, \theta, \varphi, t)$ :

$$
\begin{aligned}
& 0 \\
& f \theta, \varphi] \mathrm{h}[t-r \sqrt{\epsilon 0} \sqrt{\mu 0}]) \\
& 0
\end{aligned}
$$

Equation (33) and (34) are solutions of (8) under the influence of a Radial Gravitational field with field a gravitational constant $\mathrm{G}$ and a gravitational field intensity $\mathrm{g}$ $\left[\mathrm{m} / \mathrm{s}^{2}\right]$ :

$$
g=\mathrm{G} \frac{\mathrm{m}_{1}}{\mathrm{r}^{2}}
$$

Which acts along the radial-direction while the electromagnetic wave is also propagating in the radialdirection.

When a light beam leaves the surface of the sun, the intensity will decrease according (8). At earth, the measured intensity (Ref. [11], page 29) will be according (8): 


$$
\mathrm{I}=\frac{\mathrm{I}_{0} \mathrm{e}^{-2+\frac{\mathrm{Gm}_{1} \varepsilon_{0} \mu_{0}}{\mathrm{r}}}}{4 \pi \mathrm{r}^{2}}
$$

A beam of light represents an amount of electromagnetic energy. Which equals an amount of electromagnetic mass. This amount of electromagnetic mass is moving with the speed of light in the opposite direction of a (radial) gravitational field and gains potential energy. Because the law of conservation of energy, a part of the electromagnetic energy of the light beam has to be converted into potential energy according equations (33) and (34).

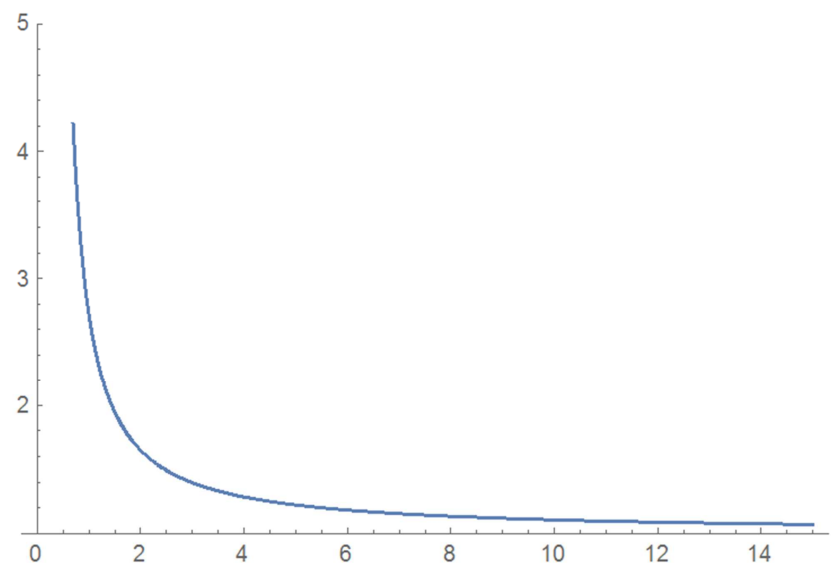

Figure 2. Electromagnetic Gravitational Conversion Term $\frac{\mathrm{G} \mathrm{m}_{1} \varepsilon_{0} \mu_{0}}{\mathrm{r}}$.

For a radius of the sun equals $695,508[\mathrm{~km}]$ and a distance from the sun to the earth of $149,600,000 \quad[\mathrm{~km}]$, the Electromagnetic Gravitational Conversion (EMGC) term equals:

$$
C_{E M G C}=\frac{\mathrm{e}^{\frac{\mathrm{Gm}_{1} \varepsilon_{0} \mu_{0}}{\mathrm{r}_{1}}}}{\mathrm{e}^{\frac{\mathrm{Gm}_{1} \varepsilon_{0} \mu_{0}}{\mathrm{r} 2}}}=\frac{\mathrm{e}^{\frac{1}{\mathrm{r}_{1}}}}{\mathrm{e}^{\frac{1}{\mathrm{r} 2}}}=4.1877534
$$

This means that the real intensity of the light at the surface of the sun is about 4 times higher than the intensity which would have been calculated in a classical way from the sunlight intensity measured on earth, due to Electromagnetic Gravitational Conversion.

Equations (33) and (34) are solutions of (8) under the influence of a Radial Gravitational field with field intensity "G" that acts along the radial-direction while the electromagnetic wave is also propagating in the same radialdirection. The electromagnetic wave is propagating with the unaltered speed of light $c=1 / \sqrt{\varepsilon_{0} \mu_{0}}$, independently of the strength $G$ of the gravitational field in the radial-direction. However, the amplitude of the electromagnetic wave becomes dependently of the gravitational intensity " $G$ " and the distance " $r$ " and changes along the radial direction due to the Electromagnetic-Gravitational Conversion term

$$
C_{E M G C}=\mathrm{e}^{\frac{\mathrm{G} \mathrm{m}_{1} \varepsilon_{0} \mu_{0}}{\mathrm{r}}}
$$

Because of the law of conservation of Energy, the electromagnetic energy of the light emitted by the sun is decreasing over a distance " $r$ " proportional with the same amount $E m G C=e^{\frac{G m_{1} \varepsilon_{0} \mu_{0}}{r}}$ as the potential energy of the electromagnetic mass of the light emitted by the sun is increasing.

\subsection{Transversal Electromagnetic Mass (Inertia)/Confined Mass (The Impact of Gravity on Light)}

In chapter 4.1.4 it has already been demonstrated that the classical concept of mass has been related to confined electromagnetic radiation. A LASER beam is an example of 2-Dimensional confinement.

It has already been demonstrated in chapter 4.2 that a LASER beam has no classical mass in the direction of propagation. In the direction of propagation an energy exchange takes place between potential energy (caused by a gravitational field in the direction of propagation) and the electromagnetic energy of the beam itself.

A Laser beam only expresses the classical property of mass in the direction transversal to the direction of propagation. For that reason, a LASER beam propagates around a source of a gravitational field like a satellite moves in a circular orbit around the earth.

We consider a beam of light approaching a strong gravitational field. (E.g. a Black Hole). According the first term in equation (8) the beam of light will follow a circular orbit around the Black Hole. The required Equilibrium will exist at the radius where the centrifugal electromagnetic inertia forces will be equal and opposite directed to the centripetal oriented gravitational forces on the electromagnetic mass.

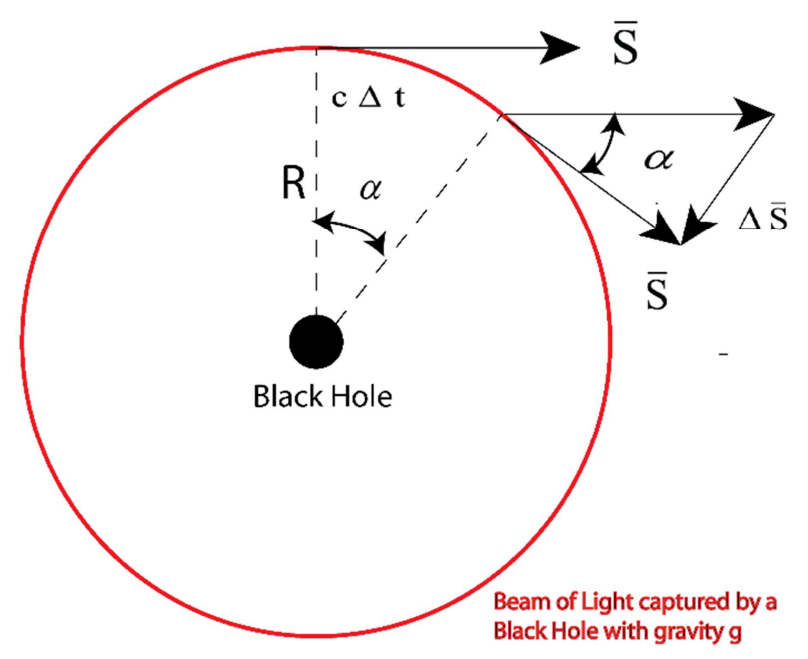

Figure 3. LASER beam around a Black Hole.

In Figure 3 "the term $\Delta \mathrm{S}$ divided by a time interval $\Delta \mathrm{t}$ " equals: 


$$
\frac{\Delta \mathrm{S}}{\Delta \mathrm{t}}=\mathrm{c} \frac{\Delta \mathrm{p}}{\Delta \mathrm{t}}=\mathrm{c} \frac{\Delta(\mathrm{m} \mathrm{v})}{\Delta \mathrm{t}}=\mathrm{cm} \frac{\Delta \mathrm{v}}{\Delta \mathrm{t}}=\mathrm{c} \mathrm{ma}
$$

In which "a" represents the radial acceleration and is part of Newton's second law of motion in the radial direction. Because the beam of light has been confined in the radial direction, it demonstrates in the radial direction the property of inertia (electromagnetic mass) and interacts with a gravitational field according Newton's second law of motion. The whole Universe is in a perfect Equilibrium. This fundamental property of Equilibrium has been extended to a 4-dimensional Hyperspace Continuum in which a perfect equilibrium persists in any of the 4 coordinate directions.

The requirement of a 4-dimensional Equilibrium (Ref. [14]) results in the outcome that the Dirac Equation is only one equation in a set of 4 equations. And that the Dirac Equation originates from an electromagnetic equation in the timeenergy domain. This new 4-Dimensional Hyperspace Equilibrium Theory opens a new door to an unexplored field of mathematical and physical challenges. This theory is a new approach in physics based on a 4-Dimensional Hyperspace Equilibrium resulting in the 4-dimensional Dirac Equation which represents the "Unification of (quantum mechanical) De Broglie Waves and Electromagnetic Waves". Solving these 4 simultaneous equations (7) offers the possibility to find answers to the fundamental questions in physics within a quantum mechanical 4-Dimensional FrameWork. Every Physical Possible Electro-Magnetic Field Configuration of Confinement has to be a solution of this fundamental 4-Dimensional Relativistic Dirac Equation (7).

Equation (7) in the Time-Energy Domain (x4) equals the well-known Quantum Mechanical Dirac Equation and in a comparable way the Schrödinger wave equation [10] at nonrelativistic velocities.

The 3 Equations (7) in the 3 spatial directions (x1, x2, x3) describe the force densities within the Electromagnetic Field. The term B-1 controls the speed of light and is comparable with Newton's second law of motion. When all the external forces acting on confined electromagnetic radiation equal zero, term B-1 represents Newton's $1^{\text {st }}$ law. The terms B-2 and B-3 control the confinement of Light for the Electric Radiation Pressure and the terms B-4 and B-5 control the confinement of Light for the Magnetic Radiation Pressure. Equation (7) represents Newton's $3^{\text {rd }}$ law expressed in electromagnetic force densities within an arbitrary electromagnetic field configuration (confined or free electromagnetic radiation at any arbitrary frequency. Equation (7) in the 3 spatial directions is an improved version of the well-known Maxwell's 4 equations.

\subsection{The Physical Properties (Inertia, Mass, Momentum, Velocity) of the Photon}

Photons are unique confinements of Electromagnetic Radiation. They are 2-dimensional confinements of electromagnetic wave packages. The theory describes this completely. Photons can never be accelerated or decelerated (in the direction of propagation) because that is only possible with confined radiation. It is possible to accelerate or decelerate photons in the direction perpendicular to the direction of propagation (directions of confinement) but it is impossible to accelerate or decelerate photons in the direction of propagation. They will always (according the theory) propagate with the speed of light and for that reason a red shift will only be possible when the gravitational field is in a direction perpendicular to the direction of propagation

As a demonstration of the power of the 4-dimensional Relativistic Dirac Equation, we observe a very basic and simple polarized laser beam with a Gaussian Intensity Division. The laser beam propagates towards the z-direction. The Electric Field "E" is oriented along the $\mathrm{x}$-axis and the Magnetic Field " $\mathrm{H}$ " is oriented along the $\mathrm{y}$-axis.

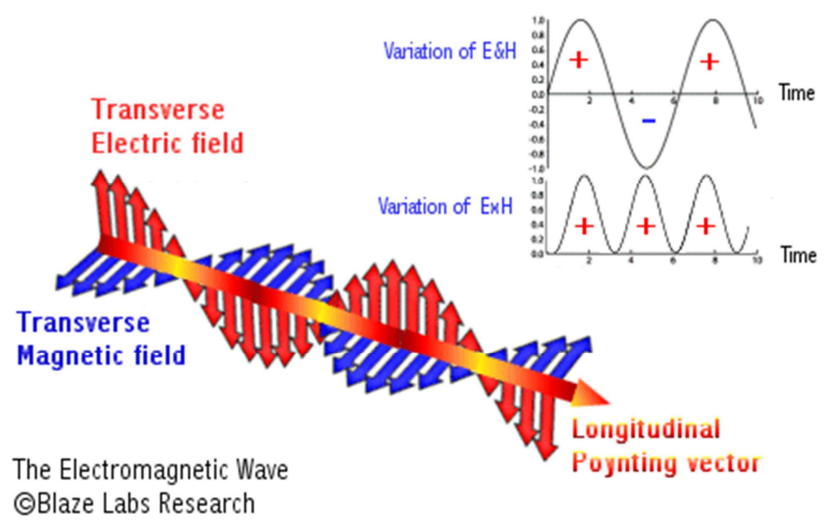

Figure 4. Polarized LASER beam.

The laser beam propagates towards the positive $\mathrm{z}$ direction:

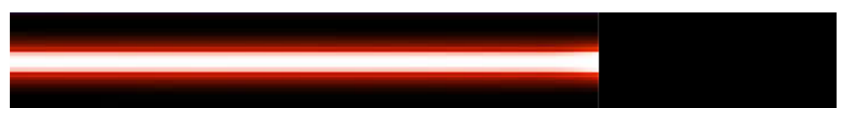

Figure 5. Polarized LASER beam (Single Shot).

The laser beam propagates towards the positive z-direction with the speed of light. That is only possible because exactly at the speed of light there will exist a "Perfect Equilibrium" between the Electromagnetic Radiation Pressure towards the positive z-direction and the inertia term of the Electromagnetic Radiation Energy (electromagnetic mass) which has been represented by the time derivative of the "Poynting Vector" in term B-1 and is oriented along the negative $\mathrm{z}$-direction.

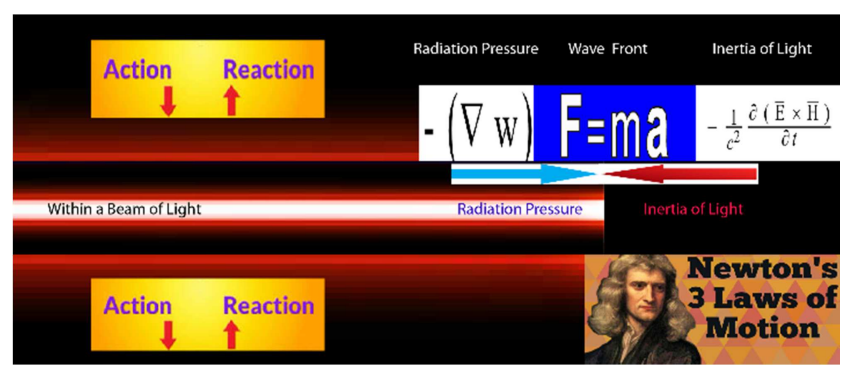

Figure 6. Newton's second Law of motion applied to a LASER Beam. 
In the Unified 4-dimensional Equilibrium always a"Perfect Equilibrium" will exist between the Electromagnetic Radiation Pressure towards the positive z-direction and the inertia term of the Electromagnetic Radiation Energy (electromagnetic mass) which has been represented by the time derivative of the "Poynting Vector" in term B-1 and is oriented along the negative $\mathrm{z}$-direction.

The inward oriented Electromagnetic Force Density in the z-direction (Electromagnetic Inertia) for every arbitrary function $\mathrm{f}(\mathrm{x}, \mathrm{y}, \mathrm{z})$ propagating with the speed of light $\mathrm{c}=1 / \sqrt{\varepsilon_{0} \mu_{0}}$ :

$$
\mathrm{f}_{z}=-\varepsilon_{0} \mu_{0} \frac{\partial \overline{\mathrm{E}} \times \overline{\mathrm{H}}}{\partial \mathrm{t}}=-2 \varepsilon_{0}^{3 / 2} \sqrt{\mu_{0}} \mathrm{f}(\mathrm{x}, \mathrm{y})^{2} \mathrm{~g}\left(\mathrm{t}-\mathrm{z} \sqrt{\varepsilon_{0} \mu_{0}}\right) \mathrm{g}^{\prime}\left(\mathrm{t}-\mathrm{z} \sqrt{\varepsilon_{0} \mu_{0}}\right)
$$

Compensates the outward oriented Electromagnetic Radiation Pressure in the z-direction for every arbitrary function $\mathrm{f}(\mathrm{x}, \mathrm{y}, \mathrm{z})$ propagating with the speed of light $\mathrm{c}=1 / \sqrt{\varepsilon_{0} \mu_{0}}$ :

$$
\mathrm{f}_{z}=-(\nabla \mathrm{w})_{z}=2 \varepsilon_{0}^{3 / 2} \sqrt{\mu_{0}} \mathrm{f}(\mathrm{x}, \mathrm{y})^{2} \mathrm{~g}\left(\mathrm{t}-\mathrm{z} \sqrt{\varepsilon_{0} \mu_{0}}\right) \mathrm{g}^{\prime}\left(\mathrm{t}-\mathrm{z} \sqrt{\varepsilon_{0} \mu_{0}}\right)
$$

The electromagnetic "Radiation Pressure" in the positive z-direction (direction of propagation) has been determined by the electromagnetic "Energy Density". The electromagnetic "Inertia Force" has been determined by the electromagnetic "Mass (Energy Density)" and the "Propagation Speed". There is only "One Exact Single Velocity" at which "Velocity" the electromagnetic "Radiation Pressure" in the in the positive z- direction perfectly counterbalances the opposite directed (negative z-direction) "Force of Inertia". That single speed has been called the "Velocity of Perfect Equilibrium" and has been called in general the "Speed of Light" and is independent of the frequency and the intensity of the electromagnetic beam of light.

$$
\text { Velocity of Perfect Equilibrium equals: } \mathrm{c}=\frac{1}{\sqrt{\varepsilon_{0} \mu_{o}}}=299,792[\mathrm{~km} / \mathrm{s}]
$$

When a laser beam (beam of light) passes a gravitational field with acceleration "g" in the (x,y) direction (Ref [5 - 8]), the radiation pressures within de the beam of light in the $(\mathrm{x}, \mathrm{y})$ plane will shift due to gravitational forces. Because according Einstein's $E=\mathrm{mc}^{2}$, the electromagnetic energy of the beam of light has electromagnetic mass, which will be attracted by the gravitational field. The shift in the radiation pressures in the $x-y$ plane due to gravitationalelectromagnetic interaction can only be compensated by the inertia forces within the $(\mathrm{x}, \mathrm{y})$ plane due to a circular orbit of the beam of light with the origin of the gravitational field as the center.

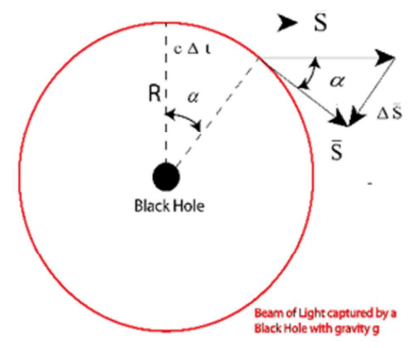

$$
\begin{aligned}
& \operatorname{Tan}(\alpha)=\frac{c \Delta \mathrm{t}}{R}=\frac{\Delta \mathrm{S}}{S} \\
& \text { Newton: } \mathrm{F} \text { (Force) }=\mathrm{m} \text { (mass) a (accelleration) } \\
& \text { in which } \left.\mathrm{f}\left(\text { force density }\left[\mathrm{N} / \mathrm{m}^{2}\right]\right) \text { and } \rho \text { (specific mass }\left[\mathrm{kg} / \mathrm{m}^{3}\right]\right) \\
& f_{\text {INERTIA }}=\frac{1}{\mathrm{c}^{2}} \frac{\Delta \mathrm{S}}{\Delta \mathrm{t}}=\frac{S}{R \mathrm{c}}=\rho \mathrm{g} \\
& \text { Einstein }\left(\mathrm{E}=\mathrm{m} \mathrm{c}^{2}\right) \\
& \frac{1}{\mathrm{c}^{2}} \frac{\Delta \mathrm{S}}{\Delta \mathrm{t}}=\frac{S}{R \mathrm{c}}=\rho \mathrm{g}=\frac{w}{c^{2}} \mathrm{~g}=\frac{\varepsilon \mathrm{E}^{2}+\mu \mathrm{H}^{2}}{2 \mathrm{c}^{2}} \mathrm{~g} \\
& \frac{E}{H}=\sqrt{\frac{\mu}{\varepsilon}} \\
& R=\frac{S}{\rho \mathrm{c} \mathrm{g}}=\frac{E^{2} \mathrm{c} \sqrt{\frac{\varepsilon}{\mu}}}{\mathrm{g} \varepsilon \mathrm{E}^{2}}=\frac{1}{\varepsilon \mu \mathrm{g}} \approx \frac{910^{16}}{g}
\end{aligned}
$$

Figure 7. Polarized LASER beam around a Black Hole.

The perfect equilibrium direction (Ref $[9,10,12,13)$ where the inertia forces due to the circular orbit of the beam of light are in a perfect balance with the attractive gravitational forces, exists at one defined radius " $\mathrm{R}$ " of the beam of light (laser beam), independent of the intensity of the beam of light and independent of the frequency of the beam of light. Only the acceleration "g" of the gravitational field determines the radius of equilibrium " $R$ "

$$
\mathrm{R} \approx \frac{910^{16}}{g}
$$

In which " $R$ " is the radius of the beam of light and " $g$ " the acceleration of the gravitational field of the "Black Hole". 
The $x-y$ plane is oriented perpendicular on the z-direction. The speed of light towards the positive $\mathrm{z}$-direction equals the speed of light (the constant "c $=300.000 \mathrm{~km} / \mathrm{s} "$ ). But the speed of light in the x-y plane has to be exactly zero (Ref. [9, $14,15)$. Else the diameter of the laser beam would become larger and larger during the propagation along the positive $\mathrm{z}$ - direction. This is only possible because the Electromagnetic confining forces B-2, B-3,B-4 and B-5 compensate exactly the outward oriented radiation pressure towards the $\mathrm{x}$ dirention and the y-direction.

The Electric Radiation Pressure has been compensated by the Coulomb Force Densities within the Laser Beam.

$$
\begin{aligned}
& \text { Coulomb's Law (B-2): } \\
& \overline{\mathrm{F}}=\overline{\mathrm{E}} \mathrm{Q} \quad(\mathrm{F} \triangleq \text { Force and } \mathrm{Q} \triangleq \text { Electric Charge }) \\
& \overline{\mathrm{f}}=\overline{\mathrm{E}} \rho \quad(\mathrm{f} \hat{=} \text { Force density and } \rho \triangleq \hat{=} \text { Charge Density }) \\
& \overline{\mathrm{f}}=\overline{\mathrm{E}} \nabla \cdot \overline{\mathrm{D}} \quad(\mathrm{D} \hat{\triangleq} \text { Dielectric Displacement }) \\
& \overline{\mathrm{f}}=\varepsilon_{0} \overline{\mathrm{E}} \nabla \cdot \overline{\mathrm{E}} \quad\left(\varepsilon_{0} \stackrel{\wedge}{=} \text { Permittivity }\right) \\
& \begin{array}{c}
\text { Energy-Time Domain } \\
\left(\mathrm{x}_{4}\right) \quad\left(\frac{\mathrm{i} \mathrm{m} \mathrm{c}}{h} \bar{\beta}+\bar{\alpha} \cdot \nabla\right) \psi+\frac{1}{c} \frac{\partial \psi}{\partial t}=0
\end{array} \\
& \text { 3-Dimensional Space Domain } \\
& \text { B-1 B-2 B-3 } \\
& \left(\begin{array}{c}
\mathrm{x}_{3} \\
\mathrm{x}_{2} \\
\mathrm{x}_{1}
\end{array}\right) \quad \begin{array}{c}
-\frac{1}{c^{2}} \frac{\partial(\overline{\mathrm{E}} \times \overline{\mathrm{H}})}{\partial t}+\varepsilon_{0} \overline{\mathrm{E}}(\nabla . \overline{\mathrm{E}})-\varepsilon_{0} \overline{\mathrm{E}} \times(\nabla \times \overline{\mathrm{E}})+ \\
+\mu_{0} \overline{\mathrm{H}}(\nabla \cdot \overline{\mathrm{H}})-\mu_{0} \overline{\mathrm{H}} \times(\nabla \times \overline{\mathrm{H}})=\overline{0}
\end{array}
\end{aligned}
$$

The Electric Radiation Pressure along the $\mathrm{x}$-axis has been compensated by the term B-2:

The inward oriented Electric Force Density in the $\mathrm{x}$ - direction for every arbitrary function $\mathrm{f}(\mathrm{x}, \mathrm{y}, \mathrm{z})$ :

$$
\mathrm{f}_{x}=\varepsilon_{0} \mathrm{E}_{x} \frac{\partial \mathrm{E}_{x}}{\partial \mathrm{x}}=\varepsilon_{0} \mathrm{f}(\mathrm{x}, \mathrm{y}) \mathrm{g}\left(\mathrm{t}-\mathrm{z} \sqrt{\varepsilon_{0} \mu_{0}}\right)^{2} \mathrm{f}^{(1,0)}(\mathrm{x}, \mathrm{y})
$$

Compensates the outward oriented Electric Radiation Pressure in the $\mathrm{x}$-direction for every arbitrary function $\mathrm{f}(\mathrm{x}, \mathrm{y}, \mathrm{z})$ :

$$
\mathrm{f}_{x}=-(\nabla \mathrm{w})_{x}=-\varepsilon_{0} \mathrm{f}(\mathrm{x}, \mathrm{y}) \mathrm{g}\left(\mathrm{t}-\mathrm{z} \sqrt{\varepsilon_{0} \mu_{0}}\right)^{2} \mathrm{f}^{(1,0)}(\mathrm{x}, \mathrm{y})
$$

The Electric Radiation Pressure along the y-axis has been compensated by the term B-3:

The inward oriented Electric Force Density in the y- direction for every arbitrary function f(x,y,z):

$$
\mathrm{f}_{y}=-\varepsilon_{0}(\overline{\mathrm{E}} \times(\nabla \times \overline{\mathrm{E}}))_{y}=-\varepsilon_{0} \mathrm{f}(\mathrm{x}, \mathrm{y}) \mathrm{g}\left(\mathrm{t}-\mathrm{z} \sqrt{\varepsilon_{0} \mu_{0}}\right)^{2} \mathrm{f}^{(0,1)}(\mathrm{x}, \mathrm{y})
$$

Compensates the outward oriented Electric Radiation Pressure in the $\mathrm{y}$-direction for every arbitrary function $\mathrm{f}(\mathrm{x}, \mathrm{y}, \mathrm{z})$ :

$$
\mathrm{f}_{y}=-(\nabla \mathrm{w})_{y}=\varepsilon_{0} \mathrm{f}(\mathrm{x}, \mathrm{y}) \mathrm{g}\left(\mathrm{t}-\mathrm{z} \sqrt{\varepsilon_{0} \mu_{0}}\right)^{2} \mathrm{f}^{(0,1)}(\mathrm{x}, \mathrm{y})
$$

This also is valid for a single photon. In the directions perpendicular to the direction of propagation, the electric radiation pressures (45) and (47) have been compensated completely by the electric interaction forces (44) and (46)

The Magnetic Radiation Pressure along the y-axis has been compensated by the term B-4:

The inward oriented Magnetic Force Density in the y-direction for every arbitrary function f(x,y,z):

$$
\mathrm{f}_{y}=\mu_{0} \mathrm{H}_{y} \frac{\partial \mathrm{H}_{y}}{\partial \mathrm{y}}=\mu_{0} \mathrm{f}(\mathrm{x}, \mathrm{y}) \mathrm{g}\left(\mathrm{t}-\mathrm{z} \sqrt{\varepsilon_{0} \mu_{0}}\right)^{2} \mathrm{f}^{(0,1)}(\mathrm{x}, \mathrm{y})
$$

Compensates the outward oriented Magnetic Radiation Pressure in the $\mathrm{y}$-direction for every arbitrary function $\mathrm{f}(\mathrm{x}, \mathrm{y}, \mathrm{z})$ : 


$$
\mathrm{f}_{y}=-(\nabla \mathrm{w})_{y}=-\mu_{0} \mathrm{f}(\mathrm{x}, \mathrm{y}) \mathrm{g}\left(\mathrm{t}-\mathrm{z} \sqrt{\varepsilon_{0} \mu_{0}}\right)^{2} \mathrm{f}^{(0,1)}(\mathrm{x}, \mathrm{y})
$$

The Magnetic Radiation Pressure along the $\mathrm{x}$-axis has been compensated by the term B-5:

The inward oriented Magnetic Force Density in the $\mathrm{x}$ - direction for every arbitrary function $\mathrm{f}(\mathrm{x}, \mathrm{y}, \mathrm{z})$ :

$$
\mathrm{f}_{x}=-\mu_{0}(\overline{\mathrm{H}} \times(\nabla \times \overline{\mathrm{H}}))_{x}=-\mu_{0} \mathrm{f}(\mathrm{x}, \mathrm{y}) \mathrm{g}\left(\mathrm{t}-\mathrm{z} \sqrt{\varepsilon_{0} \mu_{0}}\right)^{2} \mathrm{f}^{(1,0)}(\mathrm{x}, \mathrm{y})
$$

Compensates the outward oriented Magnetic Radiation Pressure in the $\mathrm{x}$-direction for every arbitrary function $\mathrm{f}(\mathrm{x}, \mathrm{y}, \mathrm{z})$ :

$$
\mathrm{f}_{x}=-(\nabla \mathrm{w})_{x}=\mu_{0} \mathrm{f}(\mathrm{x}, \mathrm{y}) \mathrm{g}\left(\mathrm{t}-\mathrm{z} \sqrt{\varepsilon_{0} \mu_{0}}\right)^{2} \mathrm{f}^{(1,0)}(\mathrm{x}, \mathrm{y})
$$

This also is valid for a single photon. In the directions perpendicular to the direction of propagation, the magnetic radiation pressures (49) and (51) have been compensated completely by the magnetic interaction forces (48) and (51).

\section{Conclusions}

The inertia of Light is not comparable with the inertia of the mechanical mass of objects like the mechanical mass we measure in mechanical experiments. The mechanical mass (inertia) of objects is isotropic and the mechanical mass of a beam of light is an anisotropic property. The mechanical mass (inertia) of objects can be determined by Newton's second law of motion. It is impossible to accelerate or to decelerate a beam of light because the speed of light is a universal constant and for that reason it is impossible to define the mass of a beam of light in the direction of propagation with Newton's second law of motion.

However, the 2-Dimensional property of inertia related to the 2-Dimensional confinement of a beam of light perpendicular to the direction of propagation can be accelerated or decelerated in the plane perpendicular to the directions of propagation like a mechanical object and fully obeys Newton's second law of motion in these directions. In a comparable way like a satellite propagates in a circular orbit around the earth, a beam of light propagates in a circular orbit around a black hole.

In the direction of propagation of a beam of light, the beam of light cannot be accelerated or decelerated because the speed of light is a universal constant. However, like a ball losing its kinetic energy (which will be transformed into potential energy) when a ball has been thrown up from the earth, a beam of light will lose its electromagnetic energy when it moves away from a black hole.

In both examples the beam of light, inertia (mass) becomes an an-isotropic property and behaves differently in the directions perpendicular to the direction of propagation compared to the direction of propagation.

The developments in scientific research are comparable with the Helix structure within a DNA molecule. When we consider the starting point of modern science 300 years ago when Newton published his famous laws representing classical mechanics, science has now reached in one way the same equation(s) of Newton. But nowadays at a higher level in which Newton's law has become 4-Dimensional (equation 5) and does not only represent classical mechanics but also represents classical electrodynamics, general relativity and quantum physics.

\section{References}

[1] Li-Xin Li; A New Unified Theory of Electromagnetic and Gravitational Interactions, Frontiers of Physics, Volume 11, Issue 6, article id. 110402 (2016); arxiv.org/abs/1511.01260.

[2] Richard Easther, Brian R Greene, Mark G Jackson and Daniel Kabat; String windings in the early universe, Journal of Cosmology and Astroparticle Physics, Volume 2005, February 2005.

[3] J. Wheeler, Phys. Rev. 97, 511 (1955).

[4] Dirk Englund, Arka Majumdar, Michal Bajcsy, Andrei Faraon, Pierre Petroff, and Jelena Vučković; Ultrafast PhotonPhoton Interaction in a Strongly Coupled Quantum DotCavity System, Phys. Rev Lett. 108, 093604, March 2012, DOI: 10.1103/PhysRevLett.108.093604.

[5] L. Filipe O. Costa, Georgios Lukes-Gerakopoulos, and Oldřich Semerák; Spinning particles in general relativity: Momentum-velocity relation for the Mathisson-Pirani spin condition; Phys. Rev. D 97, 084023 - Published 16 April 2018.

[6] Ryotaro Kase, Masato Minamitsuji, and Shinji Tsujikawa; Relativistic stars in vector-tensor theories; Phys. Rev. D 97, 084009-Published 9 April 2018.

[7] Hector O. Silva, Jeremy Sakstein, Leonardo Gualtieri, Thomas P. Sotiriou, and Emanuele Berti; Spontaneous Scalarization of Black Holes and Compact Stars from a Gauss-Bonnet Coupling; Phys. Rev. Lett. 120, 131104 (2018) - Published 30 March 2018.

[8] Jahed Abedi, Hannah Dykaar, and Niayesh Afshordi; Echoes from the abyss: Tentative evidence for Planck-scale structure at black hole horizons; Phys. Rev. D 96, 082004 (2017) Published 26 October 2017.

[9] A. Hees, T. Do, A. M. Ghez, G. D. Martinez, S. Naoz, E. E. Becklin, A. Boehle, S. Chappell, D. Chu, A. Dehghanfar, K. Kosmo, J. R. Lu, K. Matthews, M. R. Morris, S. Sakai, R. Schödel, and G. Witzel; Testing General Relativity with Stellar Orbits around the Supermassive Black Hole in Our Galactic Center; Phys. Rev. Lett. 118, 211101 (2017) Published 25 May 2017. 
[10] J. W. Vegt, A Continuous Model of Matter based on AEONs, Physics Essays, 1995, Volume 8, Number 2, 201-224 A Continuous model of Matter (https://doi.org/10.31219/osf.io/ra7ng)

[11] J. W. Vegt, Unified 4-Dimensional Hyperspace Equilibrium beyond Einstein 4-Dimensional, Kaluza-Klein 5-Dimensional and Superstring 10- and 11 Dimensional Curved Hyperspaces (https://doi.org/10.31219/osf.io/vq2a4)

[12] J. M. Maldacena, Black Holes in String Theory, Princeton University, arxiv.org/abs/hep-th/960723533.

[13] V. C. de Andrade and J. G. Pereira, Gravitational Lorentz force and the description of the gravitational interaction, Phys. Rev. D 56, 468.
[14] Volodymyr Krasnoholovets, Motion of a Relativistic Particle and the Vacuum, Physics Essays, vol 10, no 3, 1997, 407-416, arXiv: quant-ph/9903077.

[15] Donald H Kobe; Quantum power in de Broglie-Bohm theory; Journal of Physics A: Mathematical and Theoretical, Volume 40-Number 19, Published 24 April 2007.

[16] J. W. Vegt. Origin of "De Broglie Waves" (Calculations in Mathematica 11.0 in PDF format) Publisher Wolfram. https://doi.or/10.31219/osf.io/gbn4p.

[17] J. W. Vegt. Calculations in Mathematica. Solutions for an electromagnetic field under the influence of a longitudinal gravitational field. 